\title{
A Data-driven Approach for Estimating Relative Voltage Sensitivity
}

\author{
Shahab Karrari*, Michael Vollmer ${ }^{\dagger}$, Giovanni De Carne*, Mathias Noe*, Klemens Böhm ${ }^{\dagger}$ and Joern Geisbüsch* \\ ${ }^{*}$ Institute for Technical Physics (ITEP) \\ ${ }^{\dagger}$ Institute for Program Structures and Data Organization (IPD) \\ Karlsruhe Institute of Technology (KIT) \\ Karlsruhe, Germany
}

\begin{abstract}
Voltage sensitivity expresses analytically the dependency between voltage and active or reactive power. Knowing the voltage sensitivity is necessary in many power system applications, such as the Distributed Energy Resources (DER) optimal placement and control. The majority of voltage sensitivity estimation methods assume having an accurate model of the grid and only consider a balanced grid operation at the nominal point, which is not realistic. In this paper, a method based on Mutual Information (MI) is proposed, which is able to evaluate the nonlinear dependencies between two variables, in order to estimate the relative voltage sensitivity. Contrary to the existing methods, the proposed MI-based approach only requires measurements at the point of interest and does not require any grid model nor measurements from other nodes in the grid. As a use case, the optimal allocation for an Energy Storage System (ESS) in a real medium voltage network in Germany has been presented. Measurement results confirm the effectiveness of the new approach for estimating relative voltage sensitivity.
\end{abstract}

Index Terms-Voltage Sensitivity, Mutual Information, Energy Storage, Optimal Allocation.

\section{INTRODUCTION}

The high penetration level of renewable generation, Distributed Energy Resources (DER) and active loads call for advanced analysis and control methods. Such methods often require information about the voltage dependency on active and reactive power. This dependency is quantified using the voltage sensitivity formulation, which indicates how much the grid voltage can be affected if an active or reactive power change occurs. High voltage sensitivities are usually associated with weak points in the grid, as changes in power can lead to significant voltage fluctuations.

Voltage Sensitivity is most commonly used for optimal allocation of DER [1], [2], Energy Storage Systems (ESS) [3] and design of advanced grid controllers [4], [5]. A comparative study among different battery placement strategies in [6] demonstrated that voltage sensitivity-based approaches outperform other allocation algorithms in terms of post fault voltage recovery time. Controllers based on voltage sensitivity coefficients have also been effective in increasing PV hosting

This work was supported by the German Research Foundation (DFG) as part of the Research Training Group GRK 2153: Energy Status Data Informatics Methods for its Collection, Analysis and Exploitation. This work of Giovanni De Carne is supported by the Ministry of Science, Research and the Arts of the State of Baden-Wrttemberg Nr.33-7533.-30-10/67/1. capacity [4], [7]. Furthermore, voltage sensitivity coefficients have been recently used to shape the load consumption by means of new technologies, such as smart transformers [8].

The aforementioned works estimate the voltage sensitivity coefficients by linearizing the power flow equations around the operating point. This can lead to high accuracy only near that point, neglecting the changes in the operating point and influence of system nonlinearities. However, it has been shown that the voltage sensitivity can change significantly following different operating points [9]. Furthermore, a balanced grid operation is mostly assumed in such an analysis. An attempt to solve this issue has been presented analytically in [10]. Moreover, it has been shown that inverter-based DERs have an impact on the aggregated load/voltage sensitivity [11], but are often not considered in the load flow model. Consequently, the accuracy and timely estimation of voltage sensitivity coefficients is important for corrective actions, especially if the sensitivity information is included in the control action. But most importantly, such methods require a validated network admittance matrix and voltage magnitudes at all nodes, which are not always available. Thus, estimating voltage sensitivity using only measurement values and without any knowledge of the grid data has gained attention over the last years. In [12], an algorithm based on historical smart meter data is proposed. Nonetheless, this method requires measurements at different locations and historical data and load profiles to train the algorithm. A close-to-real-time approach has been proposed recently [13] to calculate these sensitivities, however, without considering changes of the voltage sensitivity during the measurement period and using a linear formulation.

In this paper, we introduce the use of a Mutual Information (MI) to estimate the relative voltage sensitivity coefficients. MI quantifies how much of the active or reactive power information can be obtained by observing the voltage profile. MI only requires the measurements gathered by a metering device such as smart meters at the point of interest and takes into account the system nonlinearities, system asymmetry and changes in the operating point. As a use case, the paper presents a practical case of finding the best location for a ESS in a real medium voltage grid in the south of Germany with more than 1200 buses. First, in order to find the top candidates for conducting measurements, the buses are ranked according 


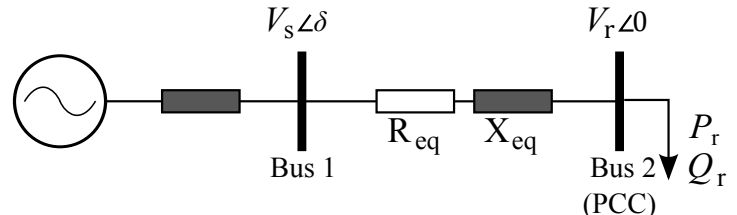

Fig. 1. Single-line diagram of a power system.

to their voltage sensitivity, obtained from the load flow model at the nominal point. Next, two weeks of measurements have been conducted for the top candidates, and the MI score is calculated for each bus. The bus with the highest MI score is suggested for ESS allocation. A comparison between the measurement results at this point and other candidates confirms the effectiveness of the proposed MI-based approach.

This paper is organized as follows. In section II voltage sensitivity is described and formulated analytically. Section III gives the definition of MI and how it reflects the dependencies between two variables. The use case of allocation of an ESS is presented in section IV. A short discussion regarding possible applications of the MI in power systems is given in section $\mathrm{V}$ and section VI concludes.

\section{Voltage Sensitivity}

To describe briefly the concept, the simplified power system as shown in Fig. 1 is considered. Between the point of common coupling (bus 2) and the main supply, there is an equivalent resistance and reactance represented by $\mathrm{R}_{\text {eq }}$ and $\mathrm{X}_{\mathrm{eq}}$, respectively. The apparent power at bus 2 can be calculated as

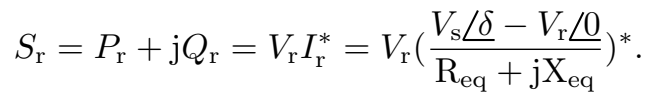

By separating the real and imaginary part of Eq. (1), the active and reactive power are calculated using

$$
\begin{aligned}
P_{\mathrm{r}} & =V_{\mathrm{r}}\left(\frac{\mathrm{R}_{\mathrm{eq}}\left(V_{\mathrm{s}} \cos \delta+V_{\mathrm{r}}-\mathrm{X}_{\mathrm{eq}} V_{\mathrm{s}} \sin \delta\right)}{\mathrm{R}_{\mathrm{eq}}{ }^{2}+\mathrm{X}_{\mathrm{eq}}{ }^{2}}\right) \\
Q_{\mathrm{r}} & =V_{\mathrm{r}}\left(\frac{\mathrm{X}_{\mathrm{eq}}\left(V_{\mathrm{s}} \cos \delta-V_{\mathrm{r}}+\mathrm{R}_{\mathrm{eq}} V_{\mathrm{s}} \sin \delta\right)}{\mathrm{R}_{\mathrm{eq}}{ }^{2}+\mathrm{X}_{\mathrm{eq}}{ }^{2}}\right) .
\end{aligned}
$$

As seen in Eq. (2) and (3), the relationship between $V_{\mathrm{r}}$ and $P_{\mathrm{r}}$ or $Q_{\mathrm{r}}$ is nonlinear. To calculate the voltage sensitivity to a small disturbance of $\Delta P_{\mathrm{r}}$ and $\Delta Q_{\mathrm{r}}$ around the operating point, we need to eliminate $\delta$, replace $P_{\mathrm{r}}$ by $P_{\mathrm{r}}+\Delta P_{\mathrm{r}}$, replace $P_{\mathrm{r}}^{2}$ by $P_{\mathrm{r}}^{2}+2 P_{\mathrm{r}} \Delta P_{\mathrm{r}}$ and neglect $\Delta P_{\mathrm{r}}^{2}$, as suggested in [14]. By doing a similar procedure with respect to $Q_{\mathrm{r}}$ and $V_{\mathrm{r}}$, the voltage sensitivity can be calculated as

$$
\begin{gathered}
\frac{\partial V_{\mathrm{r}}}{\partial P_{\mathrm{r}}} \approx \frac{\Delta V_{\mathrm{r}}}{\Delta P_{\mathrm{r}}}=\frac{\mathrm{R}_{\mathrm{eq}} V_{\mathrm{r}}^{2}+P_{\mathrm{r}}\left(\mathrm{R}_{\mathrm{eq}}{ }^{2}+\mathrm{X}_{\mathrm{eq}}{ }^{2}\right)}{V_{\mathrm{r}}\left(V_{\mathrm{s}}^{2}-2 V_{\mathrm{r}}^{2}-2\left(\mathrm{R}_{\mathrm{eq}} P_{\mathrm{r}}+\mathrm{X}_{\mathrm{eq}} Q_{\mathrm{r}}\right)\right)} \\
\frac{\partial V_{\mathrm{r}}}{\partial Q_{\mathrm{r}}} \approx \frac{\Delta V_{\mathrm{r}}}{\Delta Q_{\mathrm{r}}}=\frac{\mathrm{X}_{\mathrm{eq}} V_{\mathrm{r}}^{2}+P_{\mathrm{r}}\left(\mathrm{R}_{\mathrm{eq}}{ }^{2}+\mathrm{X}_{\mathrm{eq}}{ }^{2}\right)}{V_{\mathrm{r}}\left(V_{\mathrm{s}}^{2}-2 V_{\mathrm{r}}^{2}-2\left(\mathrm{R}_{\mathrm{eq}} P_{\mathrm{r}}+\mathrm{X}_{\mathrm{eq}} Q_{\mathrm{r}}\right)\right)} .
\end{gathered}
$$

If $V_{\mathrm{r}}$ and $V_{\mathrm{s}}$ are approximated by 1 p.u., it is clear from Eq. (4) and (5) that in case of low $\mathrm{X}_{\mathrm{eq}} / \mathrm{R}_{\mathrm{eq}}$ ratio, the voltage sensitivity to active power can be as much as or even greater than the voltage sensitivity to reactive power, which is the case in some distribution grids.

Assuming a balanced grid, for an interconnected power system with many nodes, voltage sensitivity is often estimated by linearizing the nonlinear load flow equation at the nominal operating point, that is,

$$
\left[\begin{array}{ll}
J_{P \phi} & J_{P V} \\
J_{Q \phi} & J_{Q V}
\end{array}\right]\left[\begin{array}{c}
\Delta \phi \\
\Delta V
\end{array}\right]=\left[\begin{array}{l}
\Delta P \\
\Delta Q
\end{array}\right]
$$

By inverting the load flow Jacobian matrix (J), the voltage sensitivity matrix ( $\mathrm{S}$ ) is calculated as,

$$
\left[\begin{array}{c}
\Delta \phi \\
\Delta V
\end{array}\right]=\left[\begin{array}{ll}
J_{P \phi} & J_{P V} \\
J_{Q \phi} & J_{Q V}
\end{array}\right]^{-1}\left[\begin{array}{c}
\Delta P \\
\Delta Q
\end{array}\right]=\left[\begin{array}{ll}
S_{\phi P} & S_{\phi Q} \\
S_{V P} & S_{V Q}
\end{array}\right]\left[\begin{array}{c}
\Delta P \\
\Delta Q
\end{array}\right]
$$

where $S_{V P}$ corresponds to the submatrix of $\frac{\partial V_{\mathrm{i}}}{\partial P_{\mathrm{i}}}$ (i is the bus index) or the voltage sensitivity to active power at all buses, and $S_{V Q}$ corresponds to $\frac{\partial V_{\mathrm{i}}}{\partial Q_{\mathrm{i}}}$ or the voltage sensitivity to reactive power. The voltage angles and their sensitivity are generally not a concern.

The Eq. (6) and (7) assume a balanced system. For a threephase unbalanced system, the voltage sensitivity has be to calculated for each phase with respect to the power flow of all three phases. For the jth bus:

$$
\left[\frac{\partial V_{\mathrm{j}}}{\partial P_{\mathrm{j}}}\right]=\left[\begin{array}{lll}
\frac{\partial V_{\mathrm{j} 1}}{\partial P_{1}} & \frac{\partial V_{\mathrm{j} 1}}{\partial P_{\mathrm{j} 2}} & \frac{\partial V_{\mathrm{j} 1}}{\partial P_{j 3}} \\
\frac{\partial V_{\mathrm{j} 2}}{\partial P_{2}} & \frac{\partial V_{\mathrm{j} 2}}{\partial P_{\mathrm{j} 2}} & \frac{\partial V_{\mathrm{j} 2}}{\partial P_{j 3}} \\
\frac{\partial V_{\mathrm{j} 3}}{\partial P_{\mathrm{j} 1}} & \frac{\partial V_{\mathrm{j}} 3}{\partial P_{\mathrm{j} 2}} & \frac{\partial V_{\mathrm{j} 3}}{\partial P_{\mathrm{j} 3}}
\end{array}\right]
$$

\section{MUtuAL INFORMATION}

Originating in the field of information theory, Mutual Information [15] is a number to characterize the relationship between two variables. It quantifies the expected amount of information that one attribute value, e.g., voltage, reveals about the value of another attribute, e.g., power. For instance, if two sensors always yield measurements with the same sign, regardless of absolute value, then they share (at least) one bit of information. Less formally, MI quantifies "Given the value of one attribute, how much does this help to guess the value of the other attribute?". In a sense, MI is more general than specific measures such as Pearson's Correlation Coefficient, because MI is zero if and only if the attributes are statistically independent. This capability to quantify arbitrary linear and nonlinear dependencies is the reason why datadriven approaches and Machine Learning use MI [16].

\section{A. Estimation in Continuous Domains}

MI is defined for probabilities and requires estimation if it is used for measurements, which are always limited in number. This is particularly challenging for numerical values from continuous domains with small to moderate data sizes. A well-known estimation approach called $K S G$ for its creators Kraskov, Stögbauer and Grassberger [17] sidesteps this 


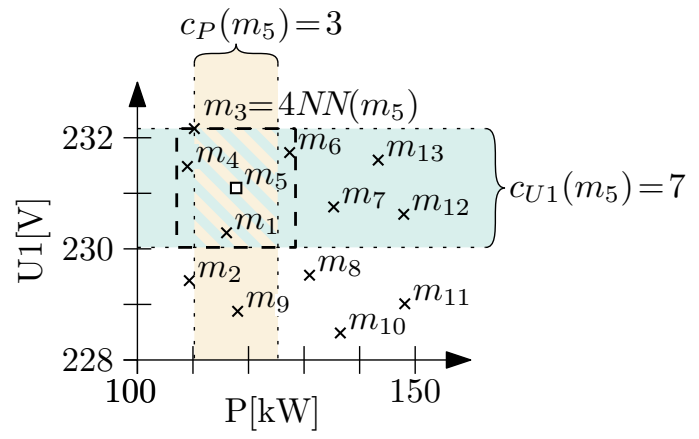

Fig. 2. Example of notation used with the KSG estimator.

chellenge by not estimating the distributions explicitly. That is, the KSG uses distances between similar measurements as substitution to distribution densities, which is fast to compute and yields good results even for small and noisy data [18].

In the following we formally define the KSG. We use Fig. 2 as an illustration of notation for one instance. Let $M=\left\{m_{1}, \ldots, m_{n}\right\}$ with $m_{i}=\left(a_{i}, b_{i}\right)$ be a collection of measurements with two attributes $A$ and $B$. In Fig. $2 M$ has 13 measurements with phase voltage $U 1$ and phase active power $P$ as attributes. For any number $k \in \mathbb{N}^{+}$, the $k$-th nearest neighbor of a measurement $m_{i}$ is $k N N\left(m_{i}\right)$ using the maxdistance, i.e., $\operatorname{dist}\left(m_{i}, m_{j}\right)=\max \left(\left|a_{i}-a_{j}\right|,\left|b_{i}-b_{j}\right|\right)$. This is shown in Fig. 2 with $m_{3}$ being the fourth nearest neighbor of $m_{5}$ with dashed lines indicating the area with points closer to $m_{5}$ than $m_{3}$. Furthermore, let $c_{A}\left(m_{i}\right)$ and $c_{B}\left(m_{i}\right)$ be the number of individual measurements for $A$ and $B$, respectively, that are no further from $m_{i}$ than $k N N\left(m_{i}\right)$. As example, in Fig. 2 there are 3 such measurements for $P$ as shown by the vertical dotted lines, i.e., $c_{P}\left(m_{5}\right)=3$. Finally, the KSG estimate is

$M I(A, B)=\psi(n)+\psi(k)-\frac{1}{k}-\frac{1}{n} \sum_{i=1}^{n} \psi\left(c_{A}\left(m_{i}\right)\right)+\psi\left(c_{B}\left(m_{i}\right)\right)$,

with the digamma function $\psi(x)=\left(\sum_{m=1}^{x-1} \frac{1}{m}\right)-\gamma$ where $\gamma \approx-0.577$ is the Euler-Mascheroni constant. We use $k=4$ as literature recommends [18] $k$ as small constant. In this paper, we call values calculated using Eq. (9) MI scores.

\section{B. Maintaining Up-To-Date Estimates in Real-Time}

Recent work [19] shows that the time required to compute Eq. (9) scales super-linearly with the number of measurements. Even when using a Sliding-Window approach, i.e., using only a fixed number of the most recent measurements, recomputing the MI score from scratch with every new measurement is often infeasible with very frequent measurements. The same work proves that individual updates of a sliding window changes only a fixed number of nearest-neighbor relationships. Utilizing this insight to quickly find the changed relationships and compute the difference induced by these changes enables faster updates of MI scores [19]. Even on commodity hardware this enables real-time monitoring for measurements in each second with a sliding window of a full day. This shows the feasibility of using MI in adaptive controllers for DER and ESS.

\section{Use CAse: ESS Allocation}

The allocation of an ESS in a real MV network in the south of Germany is presented as use case of the proposed MI-based approach. The grid operator requires to install the ESS at one of the MV/LV substations, where there is sufficient space and communication infrastructure for the ESS. The total search space includes more than 1200 substations. Here the ESS can filter short-term disturbances coming from the grid in order to protect costumers who need high power quality, and also damp disturbances coming from the LV grid.

The allocation algorithm is depicted in Fig. 3. It consists of two main steps, which are described here.

\section{A. Step 1: Finding the top candidates}

In order to select the most promising buses for conducting measurements, the buses with the highest voltage sensitivity are selected. Here the voltage sensitivity was conducted using the linearized load flow model, as doing measurements at all buses is not possible. This approach is a similar to the previous studies [1]-[3]. The grid can benefit the most from the ESS at the most sensitive points in the grid, in terms of voltage regulation, harmonics and asymmetry compensation. This part of the allocation algorithm is implemented using DIgSILENT Programming Language (DPL) in DIgSILENT PowerFactory. The algorithm selects all the $10 / 0.4 \mathrm{kV}$ substation and calculate the voltage sensitivity by linear approximation around the nominal operating point, as explained in section II. The buses with the highest sensitivity to active power changes are the top candidates for the ESS installation, as long as the following constraints are met:

1) C1: No overload conditions for power systems components (transformers, lines) should occur at the ESS rated power, for charging and discharging.

2) C2: Grid code requirements (e.g., VDE-AR-N 4105) have to remain fulfilled, i.e. the voltage limit of $3 \%$ of the nominal value should not be exceeded at ESS rated power.

3) C3: Considering 1.2 p.u. for the ESS short-circuit current, no interference with the current grid protection settings must occur.

4) C4: Reverse power flow has to be avoided.

In order to carry out a load flow analysis, the ESS is modeled as a PQ bus, in which the reactive power varies according to the $\mathrm{Q}(\mathrm{V})$ characteristics indicated in the German Grid code VDE-AR-N 4105:2018-08 (Fig. 4).

If these constraints are respected, the selected bus is chosen as measurement point for a minimum period of two weeks. This has been done for the top candidate along with the next 8 candidates.

\section{B. Step 2: MI Estimation}

For the top 9 candidates for the ESS installation, two weeks of measurements have been conducted at the grid in south 


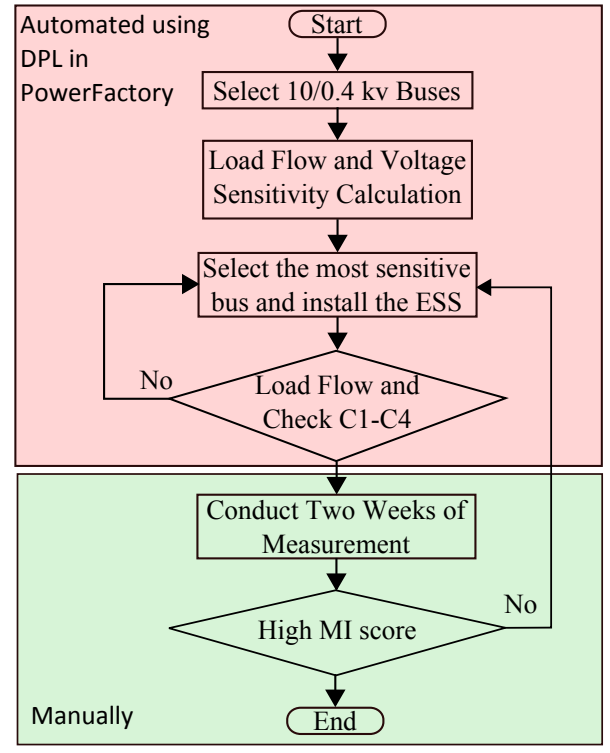

Fig. 3. The allocation algorithm for an ESS.

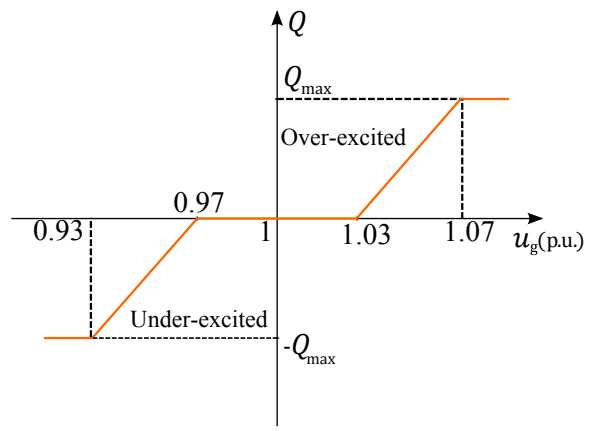

Fig. 4. The $\mathrm{Q}(\mathrm{U})$ characteristics of an ESS according to the VDE-AR-N 4105:2018-11.

Germany. These measurements are conducted using a power quality analyzer at the secondary side of $10 / 0.4 \mathrm{kV}$ substations with 1-second data resolution.

The comparison of the MI score of the measured buses are shown in Fig. 5. For each bus, the average MI score between the voltage and active and reactive power during the two weeks of the measurement period is calculated. As seen, bus 4 has the highest MI score, indicating higher relative voltage sensitivity in comparison to others. Therefore, this location is proposed for the ESS installation. Bus 5 and 2 have the lowest MI score for active and reactive power, respectively, indicating low voltage sensitivity. The results for bus 5, 7 and 8 show a higher MI score between voltage and active power than the MI score between the voltage and reactive power. This can show the resistive nature of the grid at these points.

In order to evaluate the proposed approach, the measurement results of the bus 4 and bus 5, i.e., the buses with the highest and lowest MI score, are illustrated for a period of two days with 1-minute resolution in Fig. 6. The MI results show that bus 4 has the highest MI score and therefore, the highest voltage sensitivity. This can be observed by comparing Fig. 6(a)

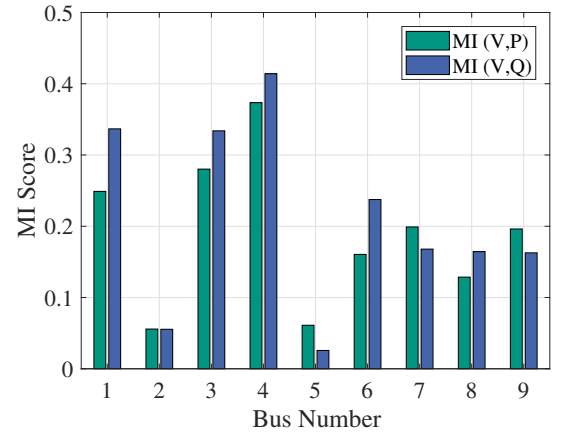

Fig. 5. Comparison of MI score for different buses.
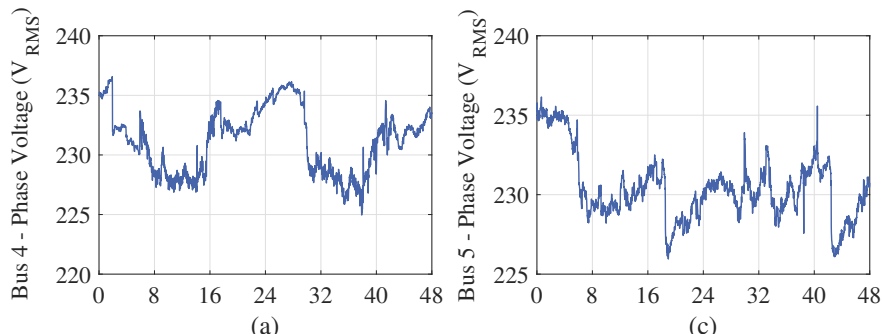

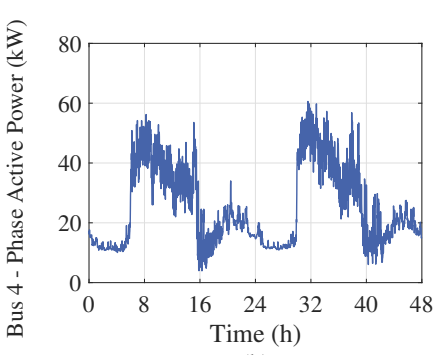

(b)

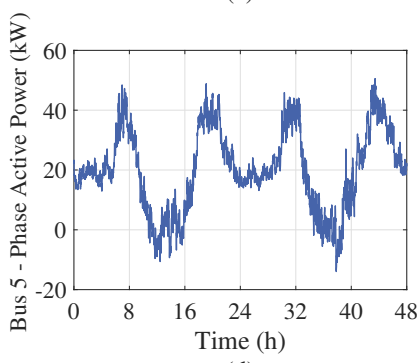

(d)
Fig. 6. Measurement results. (a) bus 4 voltage (b) bus 4 active power (c) bus 5 voltage (d) bus 5 active power.

and Fig. 6(b). As seen, the active power inversely affects the voltage, and the variables are obviously very dependant on eachother, indicating a weak point in the grid. As for the case of bus 5, with the lowest MI score, the effect of active power on voltage is not easily observable, as shown in Fig. 6(c) and Fig. 6(d), and the two variables seem very independent. This is the characteristic of a rigid point of the grid.

Unlike the load flow model, which assumes a balanced network, the real measurements reflect the asymmetries in the grid. The MI score can be calculated for every phase and between all attributes, as shown in Fig. 7. These values can reflect the values calculated analytically using Eq. (8). As seen, the MI score for the phases can be very different, which can show unbalanced operation of the grid or even unbalanced components. For instance, one can see the MI score between voltage and active power is higher than the one between voltage and reactive power for phase 1 and 3, but not for phase 2 .

\section{Discussion}

Classical methods to calculate voltage sensitivities require a validated grid model. In this paper, it has been shown that the 


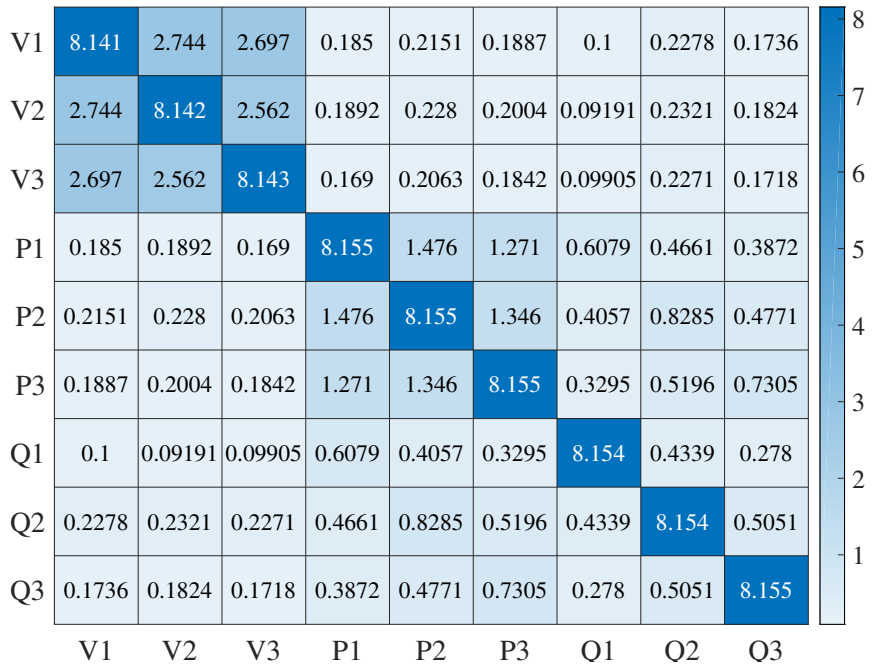

Fig. 7. Mutual Information Score for all the attributes of the three-phases of bus 7 .

MI can reflect relative voltage sensitivity coefficients and it only requires local measurement data to do that. However, an exact mapping between the per-unit MI scores and the voltage sensitivity coefficients, which has a physical meaning, requires more research and experiments. This requires synchronized measurements at all nodes in the grid together with an accurate model of the grid.

We believe that MI score has likely the potential to be used for detection of topology changes (similar to [20]), equivalent impedance estimation and improving load modeling. For instance, a significant change in the MI score can indicate grid topology changes such as islanding.

\section{CONCLUSION}

In this paper, a method based on Mutual Information is proposed for estimating the relative voltage sensitivity to active and reactive power changes. Contrary to linearized methods, such as Jacobian-based ones, MI uses only the measurement values at the point of interest, which smart meters can provide, and does not require any information from the grid model and its admittance matrix. MI captures the system nonlinearities and can also be easily be calculated for unbalanced systems and provides information on the voltage sensitivity of one phase to power changes of all phases. As use case, this work investigated the optimal allocation for ESS in a large German MV grid. Top candidates for measurements are selected based on a load flow model of the grid at the nominal operation point. Then, measurement data were collected at these candidates, and the MI scores have been calculated using this data. The bus with the highest MI has been chosen as the installation place for the ESS. Measurement results confirm that the voltage at the selected location is strongly sensitive to the active and reactive power changes, indicating high voltage sensitivity.

\section{REFERENCES}

[1] D. H. Popović, J. A. Greatbanks, M. Begović, and A. Pregelj, "Placement of distributed generators and reclosers for distribution network security and reliability," International Journal of Electrical Power and Energy Systems, vol. 27, no. 5-6, pp. 398-408, 2005.

[2] R. S. Al Abri, E. F. El-Saadany, and Y. M. Atwa, "Optimal placement and sizing method to improve the voltage stability margin in a distribution system using distributed generation," IEEE Transactions on Power Systems, vol. 28, no. 1, pp. 326-334, 2013.

[3] T. Aziz, S. R. Deeba, and Nahid-Al-Masood, "Investigation of post-fault voltage recovery performance with battery-based energy storage system in a microgrid," Proceedings of the 2016 Australasian Universities Power Engineering Conference, AUPEC 2016, pp. 1-6, 2016.

[4] S. Hashemi and J. Østergaard, "Efficient Control of Energy Storage for Increasing the PV Hosting Capacity of LV Grids," IEEE Transactions on Smart Grid, vol. 9, no. 3, pp. 2295-2303, 2018.

[5] R. Tonkoski, L. A. Lopes, and T. H. El-Fouly, "Coordinated active power curtailment of grid connected PV inverters for overvoltage prevention," IEEE Transactions on Sustainable Energy, vol. 2, no. 2, pp. 139-147, 2011.

[6] F. Ahmed, A. U. Mahin, A. Islam, and T. Aziz, "A comparative study on different placement algorithms of battery energy storage system in microgrid," in 4th International Conference on Advances in Electrical Engineering, ICAEE 2017, vol. 2018-Janua, 2018, pp. 565-569.

[7] R. Aghatehrani and A. Golnas, "Reactive power control of photovoltaic systems based on the voltage sensitivity analysis," in IEEE Power and Energy Society General Meeting. IEEE, 2012, pp. 1-5.

[8] G. De Carne, G. Buticchi, M. Liserre, and C. Vournas, "Load control using sensitivity identification by means of smart transformer," IEEE Transactions on Smart Grid, vol. 9, no. 4, pp. 2606-2615, July 2018.

[9] K. M. I. U. Ranaweera, "Energy Storage for Control of Distributed Photovoltaic Power Systems," Ph.D. dissertation, Norwegian University of Science and Technology (NTNU), 2019.

[10] K. Christakou, J. Y. Leboudec, M. Paolone, and D. C. Tomozei, "Efficient computation of sensitivity coefficients of node voltages and line currents in unbalanced radial electrical distribution networks," IEEE Transactions on Smart Grid, vol. 4, no. 2, pp. 741-750, 2013.

[11] G. De Carne, M. Langwasser, M. Ndreko, R. Bachmann, R. W. De Doncker, R. Dimitrovski, B. J. Mortimer, A. Neufeld, F. Rojas, and M. Liserre, "Which deepness class is suited for modeling power electronics?: A guide for choosing the right model for grid-integration studies," IEEE Industrial Electronics Magazine, vol. 13, no. 2, pp. 4155, June 2019.

[12] S. Weckx, R. D'Hulst, and J. Driesen, "Voltage Sensitivity Analysis of a Laboratory Distribution Grid With Incomplete Data," IEEE Transactions on Smart Grid, vol. 6, no. 3, pp. 1271-1280, 2015.

[13] C. Mugnier, K. Christakou, J. Jaton, M. De Vivo, M. Carpita, and M. Paolone, "Model-less/measurement-based computation of voltage sensitivities in unbalanced electrical distribution networks," in 19th Power Systems Computation Conference (PSCC 2016), Genoa, Italy, 2016, pp. 1-7.

[14] Y. Hase, Handbook of Power Systems Engineering with Power Electronics Applications Second Edition, 2nd ed. John Wiley \& Sons, 2013.

[15] T. M. Cover and J. A. Thomas, Elements of information theory, 2nd ed., ser. A Wiley-Interscience publication. Hoboken, NJ: WileyInterscience, 2006.

[16] H. Peng, F. Long, and C. Ding, "Feature selection based on mutual information criteria of max-dependency, max-relevance, and minredundancy," IEEE Transactions on Pattern Analysis and Machine Intelligence, vol. 27, no. 8, pp. 1226-1238, 2005.

[17] A. Kraskov, H. Stögbauer, and P. Grassberger, "Estimating mutual information," Phys. Rev. E, vol. 69, 2004.

[18] S. Khan, S. Bandyopadhyay, A. R. Ganguly, S. Saigal, D. J. Erickson, V. Protopopescu, and G. Ostrouchov, "Relative performance of mutual information estimation methods for quantifying the dependence among short and noisy data," Phys. Rev. E, vol. 76, 2007.

[19] M. Vollmer, I. Rutter, and K. Böhm, "On complexity and efficiency of mutual information estimation on static and dynamic data," in International Conference on Extending Database Technology (EDBT '18), 2018, pp. 49-60.

[20] K. Soumalas, G. Messinis, and N. Hatziargyriou, "A data driven approach to distribution network topology identification," 2017 IEEE Manchester PowerTech, Powertech 2017, 2017. 